\title{
Acute necrotizing pancreatitis as a fatal complication following DC Bead transcatheter arterial chemoembolization for hepatocellular carcinoma: A case report and review of the literature
}

\author{
TAKASHI YAMAGUCHI ${ }^{1,2}$, TOSHIHITO SEKI ${ }^{1,2}$, ATSUSHI KOMEMUSHI ${ }^{3}$, \\ KANEHIKO SUWA ${ }^{1,2}$, RINAKO TSUDA ${ }^{1,2}$, RYOSUKE INOKUCHI ${ }^{1,2}$, MIKI MURATA ${ }^{1,2}$, \\ MICHIKO YUKI $^{4}$, YOKO HARIMA ${ }^{3}$ and KAZUICHI OKAZAKI ${ }^{1}$ \\ ${ }^{1}$ Department of Gastroenterology and Hepatology, Kansai Medical University, Hirakata, Osaka 573-1010; \\ ${ }^{2}$ Liver Disease Center and ${ }^{3}$ Department of Radiology, Kansai Medical University Medical Center, Moriguchi, \\ Osaka 570-0074; ${ }^{4}$ Department of Pathology, Kansai Medical University, Hirakata, Osaka 573-1010, Japan
}

Received May 31, 2018; Accepted July 31, 2018

DOI: $10.3892 / \mathrm{mco} .2018 .1690$

\begin{abstract}
Transcatheter arterial chemoembolization (TACE) is one of the most effective palliative measures for patients with inoperable hepatocellular carcinoma (HCC). Acute pancreatitis is a rare but well-known complication following TACE. We herein present the case of a patient with HCC who developed fatal complications (acute necrotizing pancreatitis and upper gastrointestinal ulcers) after TACE with DC Beads. The patient was diagnosed with HCC in segments 4 and 8, measuring $\sim 70 \mathrm{~mm}$ in greatest diameter, and he was treated by TACE. Hepatic arteriography revealed replacement of the origin of the right hepatic artery to the superior mesenteric artery, while the left hepatic artery originated from the celiac artery. After selection of the segmental arterial branches feeding the tumor, $2 \mathrm{ml}$ of 100-300- $\mu$ m-sized DC Beads loaded with $150 \mathrm{mg}$ epirubicin were injected through the microcatheter. The patient complained of abdominal pain after the TACE procedure. Upper gastrointestinal endoscopy revealed longitudinal ulcers from the esophagus to the duodenum, and contrast-enhanced computed tomography revealed swelling of the pancreas and focal areas of low density in the pancreatic body, suggesting necrosis. The patient developed respiratory insufficiency, renal failure and sepsis, and finally succumbed to the complications 54 days after the procedure, despite general management of the acute pancreatitis. An autopsy revealed that the main cause of the patient's death was extensive pancreatic necrosis due to a gastroduodenal artery embolism after TACE
\end{abstract}

Correspondence to: Dr Takashi Yamaguchi, Department of Gastroenterology and Hepatology, Kansai Medical University, 2-5-1 Shinmachi, Hirakata, Osaka 573-1010, Japan

E-mail: yamaguct@hirakata.kmu.ac.jp

Key words: hepatocellular carcinoma, transcatheter arterial chemoembolization, DC Beads, acute pancreatitis, complications with DC Beads. Therefore, it is crucial for treating physicians to be aware of this complication following TACE with DC Beads, particularly in patients with anatomical variations.

\section{Introduction}

Transcatheter arterial chemoembolization (TACE) is considered to be one of the most effective palliative measures for patients with inoperable hepatocellular carcinoma (HCC). Recently, TACE using the drug-eluting embolic microsphere DC Beads (Biocompatibles, Farnham, UK) loaded with doxorubicin resulted in better objective response rates with fewer systemic complications compared with those in patients who underwent conventional TACE procedures (1). Chemoembolization with drug-eluting beads has the potential advantage of increasing local, effective drug delivery, while reducing the systemic bioavailability of the chemotherapeutic drug.

Rarely, extrahepatic organs may incur ischemic damage, such as acute cholecystitis (2), necrosis of the epigastric skin (3), interstitial pneumonitis (4), gastrointestinal tract ulceration (5) and acute pancreatitis (6-9). The proposed mechanism underlying these complications is inadvertent embolization through the collateral vessels or regurgitation of the chemotherapeutic agent into the arteries of other organs. However, only few reports have confirmed the presence of embolic material in damaged organs on pathological examination (3).

We herein present the case of a patient with HCC who developed acute necrotizing pancreatitis as a fatal complication following TACE with DC Beads. To the best of our knowledge, this is the first report of an autopsy case due to acute necrotizing pancreatitis after TACE using DC Beads.

\section{Case report}

An 85-year-old male patient with alcohol-related cirrhosis (Child-Pugh class A) was admitted to the Kansai Medical University Medical Center (Moriguchi, Japan) in April 2014 
for treatment of relapsed HCC. The patient had received three sessions of TACE with lipiodol (conventional TACE) to treat HCC in segments 4 and 8 , measuring $\sim 70 \mathrm{~mm}$ in greatest diameter. After the last TACE procedure 3 months earlier, contrast-enhanced computed tomography (CT) indicated that the tumor exhibited early arterial phase enhancement. The laboratory findings were as follows: Hemoglobin, $11.5 \mathrm{~g} / \mathrm{dl}$; white blood cell count, 3,600/ $\mu$; p platelet count, $174,000 / \mu 1$; albumin, $4.0 \mathrm{~g} / \mathrm{dl}$; aspartate aminotransferase (AST), $25 \mathrm{IU} / \mathrm{l}$; alanine transaminase (ALT), $10 \mathrm{IU} / 1$; alkaline phosphatase, 333 IU/l; $\gamma$-glutamyltransferase, $28 \mathrm{IU} / 1$; total bilirubin, $0.6 \mathrm{mg} / \mathrm{dl}$; prothrombin time international normalized ratio (INR), 1.08; $\alpha$-fetoprotein, $3.4 \mathrm{ng} / \mathrm{ml}$; and protein induced by vitamin $\mathrm{K}$ absence/antagonist-II, 450 AU/1. Given the diagnosis of local recurrence, TACE was performed. Hepatic arteriography demonstrated replacement of the origin of the right hepatic artery to the superior mesenteric artery, while the left hepatic artery originated from the celiac artery (Fig. 1A and B). CT during hepatic arteriography demonstrated hypervascular tumors in segments 4 and 8 , which were fed by the right hepatic artery and the medial segmental artery arising from the left hepatic artery, respectively (Fig. 1C and D). After selection of the segmental arterial branches feeding the tumors, $2 \mathrm{ml}$ of 100-300- $\mu \mathrm{m}$-sized DC Beads loaded with $150 \mathrm{mg}$ epirubicin were injected through the microcatheter, and successful embolization was achieved with complete stasis of blood flow at the level of the segmental arteries. Another arteriography performed after TACE showed disappearance of blood flow within the peripheral branches of the hepatic artery, but the flow through the gastroduodenal artery (GDA) and its branches remained intact. A few hours after the procedure, the patient complained of abdominal pain and vomited a small amount of blood, and his laboratory results revealed increased levels of AST (267 IU/l) and ALT (81 IU/l). The patient's symptoms were treated conservatively with hydration, pain relief and fever control, and they were consistent with post-embolization syndrome. Three days after the procedure, the patient underwent pan-endoscopy of the upper gastrointestinal tract and contrast-enhanced CT. The liver enzyme levels were found to be drastically increased, with an AST level of 2,538 IU/1 and an ALT level of $1,161 \mathrm{IU} / \mathrm{l}$. The white blood cell count $(5,100 / \mu \mathrm{l})$ C-reactive protein $(31.7 \mathrm{mg} / \mathrm{dl})$ and serum amylase $(865 \mathrm{IU} / \mathrm{l})$ levels were also elevated. The contrast-enhanced CT revealed swelling of the pancreas and focal areas of low density in the pancreatic body, suggesting necrosis (Fig. 2A), whereas upper gastrointestinal endoscopic examination revealed longitudinal ulcers from the esophagus to the duodenum (Fig. 3A). The patient was treated for 2 weeks with general management of acute pancreatitis, including pain control, hydration, fasting and total parenteral nutrition. However, the follow-up abdominal CT revealed peripancreatic fluid collection, with a thick, enhancing wall, suggesting necrotizing pancreatitis and an infected pseudocyst (Fig. 2B); the follow-up upper gastrointestinal endoscopy revealed purulent discharge from the duodenum (Fig. 3B). Drainage or surgical resection was not performed due to the poor general condition of the patient. $\mathrm{He}$ developed respiratory insufficiency, renal failure and sepsis, and eventually succumbed to the complications 54 days after the procedure. An autopsy was conducted with the consent of the patient's family.
The autopsy revealed necrosis of most of the hepatic tumor and extensive pancreatic necrosis. The peripancreatic tissue was also extensively necrotized, and the pancreatic head displayed hemorrhagic necrosis (Fig. 4A), involving both acinar and ductal cells, with an adjacent intense acute inflammatory reaction. In the hepatic arteries and the GDA, as well as the renal arteries, there was detection of scattered DC Beads (Fig. 4B). According to the pathologist, the patient's cause of death was extensive pancreatic necrosis due to GDA embolism after TACE with DC Beads.

\section{Discussion}

Acute pancreatitis is a rare yet well-known complication after TACE. It occurs in $\sim 1.7-2 \%$ of all patients following selective and superselective liver tumor embolization (6). This complication results from a retrograde injection of the chemotherapeutic, embolizing agent into the pancreatic arteries, giving rise to ischemic pancreatitis (8). To prevent this complication, it is important to reduce the backflow of embolic material by placing the catheter tip as close to the distal branches of the hepatic artery as possible, and embolic materials should be injected carefully to avoid regurgitation (7). From the variant arterial anatomy aspect, when numerous vessels arise from a common trunk with early bifurcation, this may affect the probability of developing ischemic injury and the severity of the injury. Our patient exhibited replacement of the origin of the right hepatic artery to the superior mesenteric artery. The left hepatic and left gastric arteries originated from the common hepatic artery in a linear manner (Fig. 1A). These anatomical characteristics are a cause of regurgitation; however, TACE procedures may be safely completed with selective, slow, steady injection into the left medial hepatic artery under fluoroscopic monitoring. It is also possible that there is an association between the frequency of this complication and the type of particles used for the embolization, the volume of particles (6), or the size of the tumor (10). In addition, the procedure must be performed with caution, as in patients with advanced atherosclerosis, iatrogenic dissection of the GDA during the procedure may cause acute pancreatitis from ischemia, since anastomosis of the superior pancreaticoduodenal artery and the superior mesenteric artery may be ineffective. However, vascular injury was not observed on pathological examination in the present case. The microscopic findings of the pancreas with the presence of foreign body material confirmed that infusion of DC Beads through the GDA may cause pancreatitis.

Post-TACE gastric lesions are also due to the backflow of embolic materials into the gastric artery and a subsequent decrease in gastric mucosal blood flow, which may cause complications such as gastric erosion or ulceration. Certain anatomical variations, such as the right gastric artery branching distally from the hepatic artery proper or from its branch or the accessory left gastric artery arising from the left hepatic artery, are most likely to be associated with a high incidence of post-TACE gastric lesions (5).

In conclusion, we herein report the case of a patient with HCC who developed the fatal complication of acute necrotizing pancreatitis following TACE with DC Beads. To the best of our knowledge, this is the first report of an autopsy case due to acute necrotizing pancreatitis after TACE 

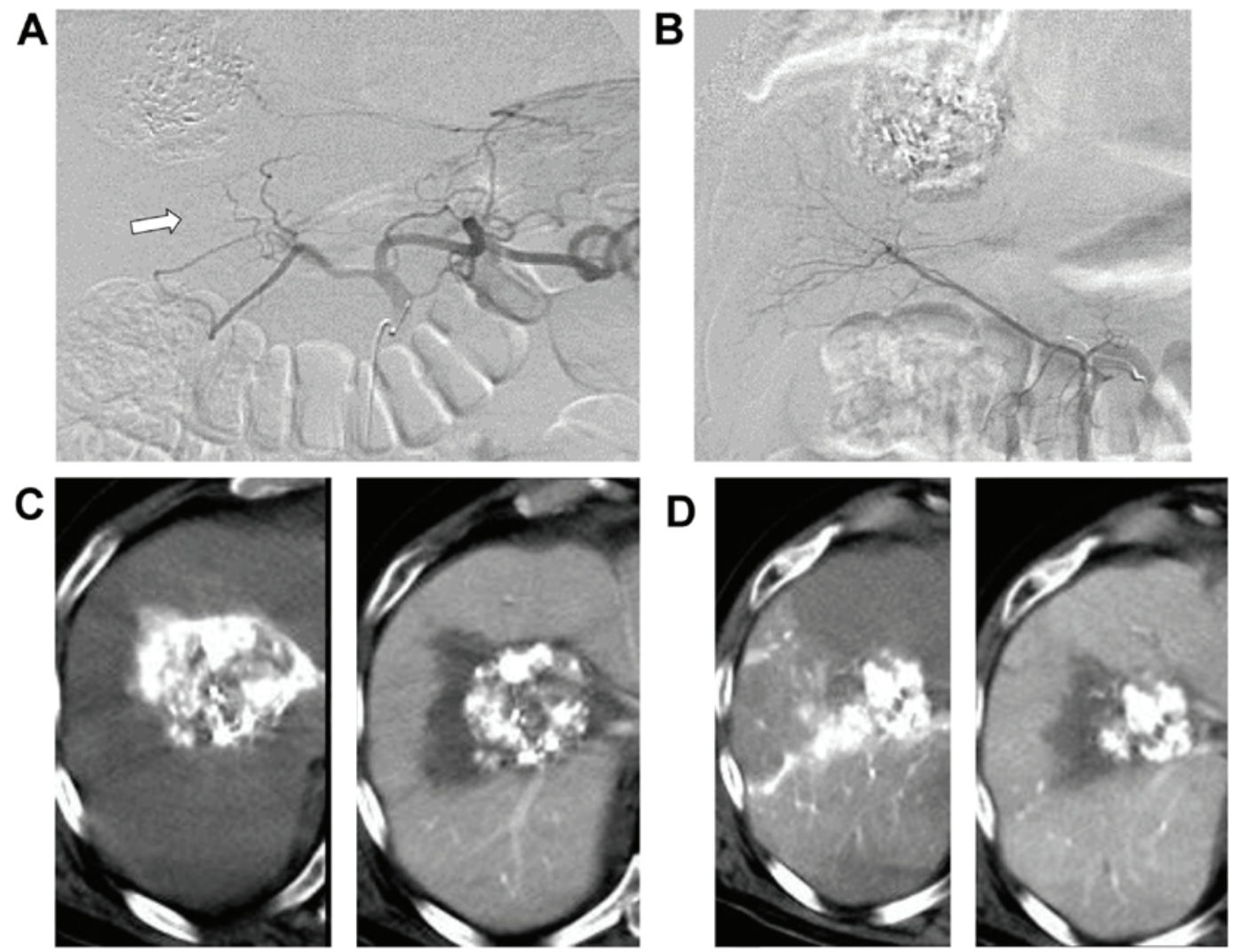

Figure 1. Celiac arteriography (A and C) and superior mesenteric arteriography (B and D) were performed. (A) The left hepatic artery that originates from the celiac artery gave rise to the medial segmental artery (arrow). (B) Angiography revealed complete displacement of the origin of the right hepatic artery to the superior mesenteric artery. (C and D) Computed tomography (CT) during hepatic arteriography (CTHA, left panel) and CT during arterial portography (CTAP, right panel) was performed. The CTAP image shows a well-defined, round perfusion defect in the right lobe of the liver. The CTHA image shows marked enhancement of the mass.
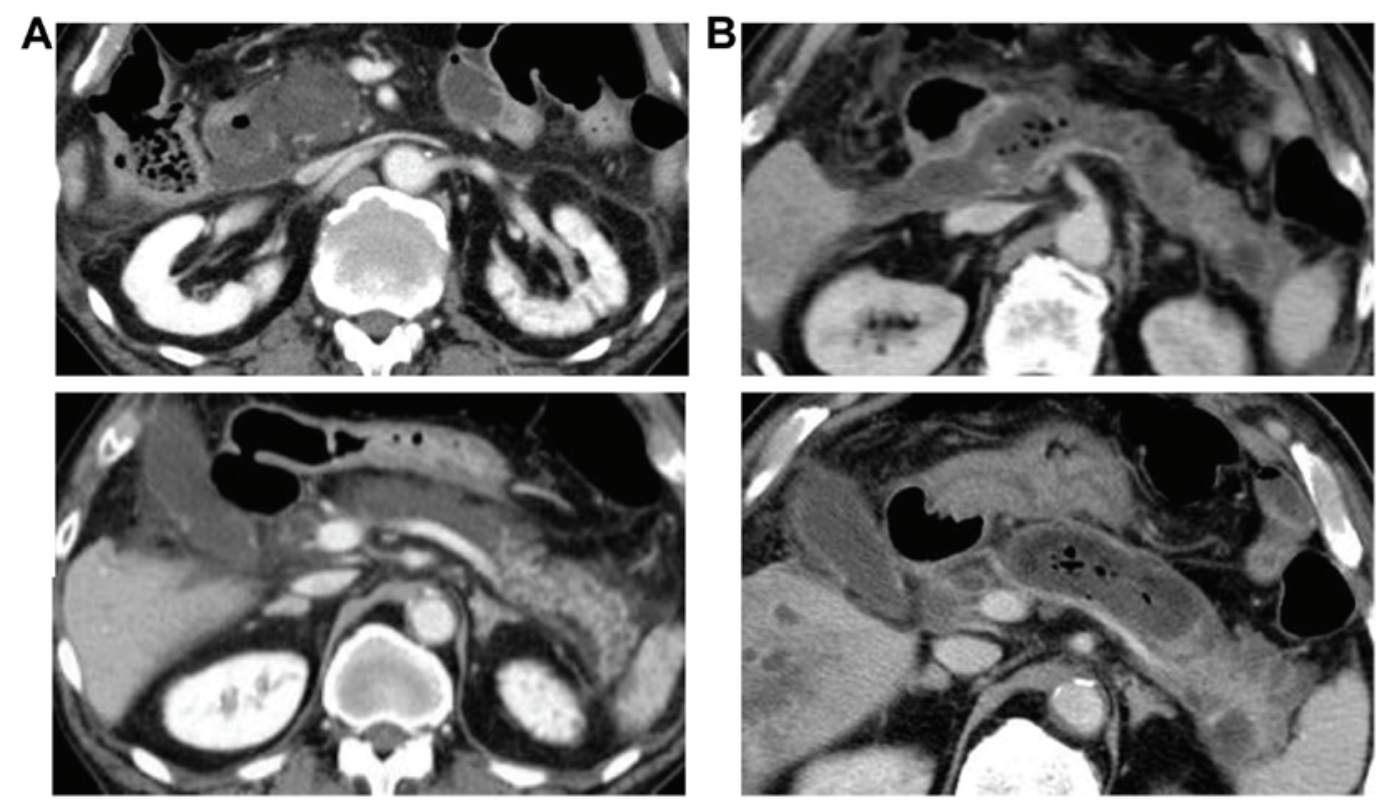

Figure 2. Axial abdominal computed tomography (CT) at the level of the pancreatic head (upper panel) and the pancreatic body (lower panel). (A) Abdominal CT image 3 days after TACE showed pancreatic swelling and a low-density area in the pancreatic head and body, suggesting necrosis. (B) Abdominal CT image 39 days after TACE showed necrotizing pancreatitis with an infected pseudocyst. TACE, transcatheter arterial chemoembolization.

using DC Beads. Although the advantage of TACE with DC Beads is fewer symptoms of post-embolization syndrome compared with lipiodol, DC Beads are hardly visible under fluoroscopic monitoring; therefore, it is difficult to identify 

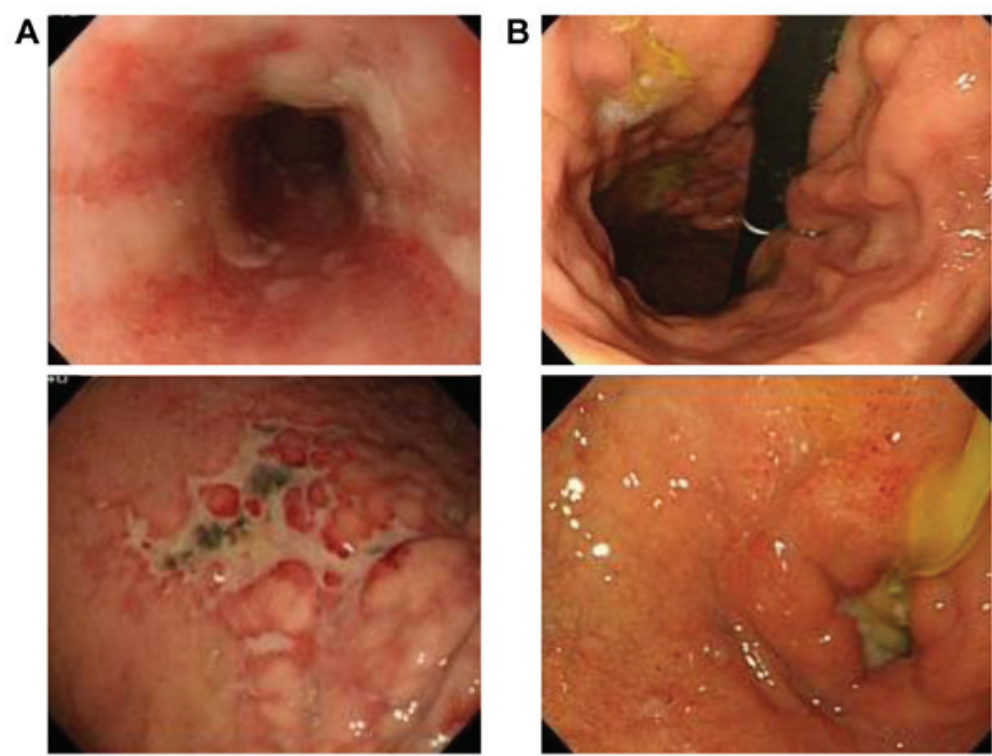

Figure 3. (A) Endoscopic appearance of the patient's esophagus (upper panel) and stomach (lower panel) 3 days after TACE. (B) Endoscopic appearance of the patient's stomach (upper panel) and duodenum (lower panel) 42 days after TACE.
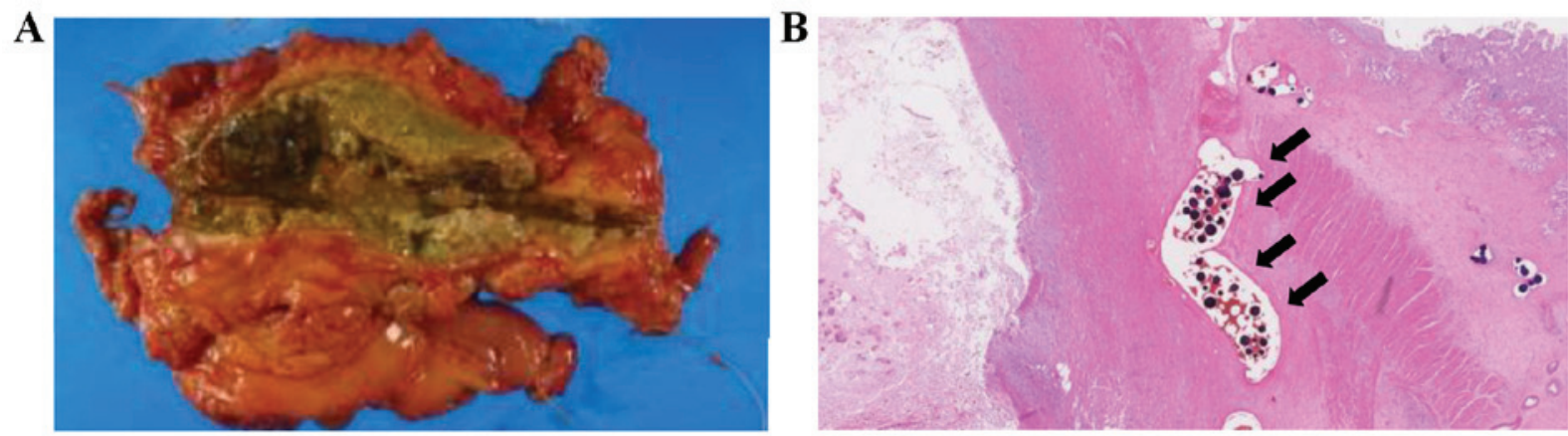

Figure 4. (A) Macroscopic appearance of the pancreas. (B) Microscopic image of the pancreas revealed the presence of DC Bead particles (arrows).

the regurgitation of the DC Beads during the procedure. Awareness of the complications following TACE with DC Beads is crucial, particularly in patients with anatomical variations.

\section{Acknowledgements}

Not applicable.

\section{Funding}

No funding was received.

\section{Availability of data and materials}

All data generated or analyzed during the present study are included in this published article.

\section{Authors' contributions}

TY and TS designed the study. TY wrote the initial draft of the manuscript. AK, RT, RI, MM and MY contributed to the analysis and interpretation of data. All authors revised the manuscript critically for important intellectual content and approved the final version of the manuscript.

\section{Ethics approval and consent to participate}

The patient's family provided written informed consent for the autopsy.

\section{Consent for publication}

The patient's family provided written informed consent for publication of the present study.

\section{Competing interests}

The authors declare that they have no competing interests.

\section{References}

1. Lammer J, Malagari K, Vogl T, Pilleul F, Denys A, Watkinson A, Pitton M, Sergent G, Pfammatter T, Terraz S, et al; PRECISION V Investigators: Prospective randomized study of doxorubicineluting-bead embolization in the treatment of hepatocellular carcinoma: Results of the PRECISION V study. Cardiovasc Intervent Radiol 33: 41-52, 2010. 
2. Karaman B, Battal B, Ören NC, Üstünsöz B and Yağci G: Acute ischemic cholecystitis after transarterial chemoembolization with drug-eluting beads. Clin Imaging 36: 861-864, 2012.

3. Kim HY, Bae SH, Park CH, Song MJ, Choi JY, Yoon SK, Jung ES and Chun HJ: Supraumbilical subcutaneous fat necrosis after transcatheter arterial chemoembolization with drug-eluting beads: Case report and review of the literature. Cardiovasc Intervent Radiol 36: 276-279, 2013.

4. Aladdin M and Ilyas M: Chemoembolization of hepatocellular carcinoma with drug-eluting beads complicated by interstitial pneumonitis. Semin Intervent Radiol 28: 218-221, 2011.

5. Leung TK, Lee CM and Chen HC: Anatomic and technical skill factor of gastroduodenal complication in post-transarterial embolization for hepatocellular carcinoma: A retrospective study of 280 cases. World J Gastroenterol 11: 1554-1557, 2005.

6. López-Benítez R, Radeleff BA, Barragán-Campos HM, Noeldge G, Grenacher L, Richter GM, Sauer P, Buchler M, Kauffmann G and Hallscheidt PJ: Acute pancreatitis after embolization of liver tumors: Frequency and associated risk factors. Pancreatology 7: 53-62, 2007.
7. Bae SI, Yeon JE, Lee JM, Kim JH, Lee HJ, Lee SJ, Suh SJ, Yoon EL, Kim HR, Byun KS, et al: A case of necrotizing pancreatitis subsequent to transcatheter arterial chemoembolization in a patient with hepatocellular carcinoma. Clin Mol Hepatol 18: 321-325, 2012.

8. Alcívar-Vásquez JM, Ontanilla-Clavijo G, Ferrer-Ríos MT and Pascasio-Acevedo JM: Acute necrotizing pancreatitis after transarterial chemoembolization of hepatocellular carcinoma: An unusual complication. Rev Esp Enferm Dig 106: 147-149, 2014.

9. Krishnamurthy P, Brown M, Agrawal S and Short RF: Acute pancreatitis as a complication of trans-arterial chemoembolization of hepatocellular cancer-case report and review of literature. J Gastrointest Oncol 8: E26-E30, 2017.

10. Malagari K, Pomoni M, Spyridopoulos TN, Moschouris H, Kelekis A, Dourakis S, Alexopoulou E, Koskinas J, Angelopoulos M, Kornezos J, et al: Safety profile of sequential transcatheter chemoembolization with DC Bead ${ }^{\mathrm{TM}}$ : Results of 237 hepatocellular carcinoma (HCC) patients. Cardiovasc Intervent Radiol 34: 774-785, 2011. 